\title{
Methodische und klinische Evaluation einer neuen enzymimmunologischen Methode zur Bestimmung des Thyroxin bindenden Globulins (TBG) und des $\mathrm{T}_{4} / \mathrm{TBG}-\mathrm{Quotienten:}$
}

\section{Eine multizentrische Studie}

Von Anne-Ch. Kessler

Boehringer Mannheim GmbH, Mannheim und Tutzing

K. H. Rudorff

Gemeinschaftskrankenhaus, Herdecke

M. Oellerich

Institut für Klinische Chemie der Medizinischen Hochschule, Hannover .

K. Horn

Medizinische Klinik Innenstadt der Universität München

J. Mattersberger

Boehringer Mannheim GmbH, Mannheim und Tutzing

R. D. Hesch

Endokrinologische Abteilung der Medizinischen Hochschule, Hannover

und Beteiligte:

C. Bernutz, Medizinische Klinik Innenstadt der Universität München

S. L. Braun, Klinikum Großhadern, München

E. Dingeon, Centre Hospitalier, Chambéry

G. Galvan, Landeskrankenanstalten, Salzburg

H. Haindl, Medizinische Hochschule, Hannover

$K$. Hengst, Universitätsklinik, Münster

S. C. Kampf, Marienkrankenhaus, Hamburg

K. Kleesiek, Universitätsklinik, Aachen

F. Rovelli, Ospedale Monza

F. Schulz, Universitätsklinik, Frankfurt

W. Vogt, Klinikum Großhadern, München

H. Wagner, Universitätsklinik, Münster

(Eingegangen am 1. Juni/24 Oktober 1984)

Zusammenfassung: Zur Bestimmung des Thyroxin bindenden Globulins (TBG) wurde ein heterogener Enzymimmunoassay entwickelt und in 12 Laboratorien evaluiert. Der Test beruht auf dem Kompetitionsprinzip und setzt mit anti-TBG Antikörper (Ziege) beschichtete Polystyrolröhrchen als Festphase ein. Über den 
Konzentrationsbereich von 4-40 mg/l TBG wurden bei Präzision in der Serie Variationskoeffizienten von 1.4-8.9\% und von Tag zu Tag von 2,9-8,6\% gemessen. Unter Verwendung einer chemisch hochreinen TBGPräparation als Standard ergaben sich im Methodenvergleich zu verschiedenen TBG-Radioimmunoassays mit Enzymun-Test ${ }^{(3)}$ TBG durchschnittlich $30 \%$ niedrigere Ergebnisse. In einer umfangreichen Referenzwertermittlung fand sich in dem normal verteilten Kollektiv schilddrüsengesunder Kontrollpersonen zwischen 18 und 50 Jahren ein Median von 14,33 mg/l TBG, 95\% aller Werte lagen zwischen 9,6-18,5 mg/1 TBG. Der Medianwert bei Frauen von $14,5 \mathrm{mg} / \mathrm{l}$ TBG lag signifikant höher als bei Männern mit 13,4 mg/l TBG. Hyperthyreote Patienten lagen im Referenzbereich, während hypothyreote Patienten erhöhte TBG Konzentrationen zeigten. Bei Frauen unter Östrogentherapie (Median: 22,2 mg/1 TBG) und in der Schwangerschaft (Median: $28.5 \mathrm{mg} / \mathrm{l} \mathrm{TBG)}$ wurden stark erhöhte TBG-Konzentrationen gemessen. Die Quotienten $\mathrm{T}_{4} / \mathrm{TBG}$ ermöglichen eine differenzierte Zuordnung zu euthyreoten (Median: 4,9), hyperthyreoten (Median: 11,3) und hypothyreoten (Median: 1,0) Stoffwechsellagen. In der Schwangerschaft liegen die Quotienten (Median: 3,1) im Vergleich zu den Kontrollpersonen signifikant niedriger.

Methodological and clinical evaluation of a new enzyme immunological method for the determination of thyroxine binding globulin $(T B G)$ and the $T_{4} / T B G$ ratio

Summary: A heterogeneous enzyme immunoassay for the determination of thyroxine binding globulin (TBG) was developed and assessed in clinical trials in 12 laboratories. The assay is based on the competition principle and employs plastic tubes coated with goat anti-TBG. CV's between $1.4-8.9 \%$ for intra-assay precision and $2.9-8.6 \%$ for inter-assay precision were found over the concentration range of $4-40 \mathrm{mg} / \mathrm{l}$ TBG. In comparative studies using highly purified TBG as standard, values with Enžymun-Test ${ }^{\circledR}$ TBG were found to be on average $30 \%$ lower than those obtained by various TBG-RIAs. A broad-base study, to determine reference values, was carried out on a group of control persons 18 to 50 years old without previous history of thyroid disease. This study revealed a median of $14.33 \mathrm{mg} / \mathrm{l} \mathrm{TBG}$, with $95 \%$ of all values between 9.6 and $18.5 \mathrm{mg} / \mathrm{l} \mathrm{TBG}$. The median in women of $14.5 \mathrm{mg} / \mathrm{l} \mathrm{TBG}$ was significantly higher than in men $(13.4 \mathrm{mg} / \mathrm{l} \mathrm{TBG})$. TBG values in hyperthyroid patients were within the reference range while those in hypothyroid individuals were elevated. Highly elevated TBG values were seen in women receiving oestrogen (median: $22.2 \mathrm{mg} / \mathrm{l} \mathrm{TBG)}$ and in pregnant women (median: $28.5 \mathrm{mg} / \mathrm{l} \mathrm{TBG}$ ). The $\mathrm{T}_{4} / \mathrm{TBG}$ ratios made it possible to distinguish between euthyroid, hyperthyroid and hypothyroic subjects (median: 4.9, 11.3 and 1.0, respectively). These ratios were significantly lower in pregnant women (median: 3.1 ) than in the control persons.

\section{Einführung}

Das Thyroxin bindende Globulin (TBG) ist das Transportprotein für Iodthyronine mit der höchsten Affinität (1). Seine Aminosäurenzusammensetzung ist bekannt, obwohl sie in unterschiedlichen Arbeiten verschieden angegeben wird $(2,3)$. Seine Aminosäuresequenz ist nur teilweise aufgeklärt, da genügende Mengen an hochreinem TBG für solche Analysen bislang nicht vorlagen (4). Trotz dieses Mangels ist die physiko-chemische Funktion von TBG hinreichend untersucht $(1-7)$. Die radioimmunchemische Bestimmung von TBG hat in den letzten Jahren immer mehr zu einer wertvollen Bereicherung in der Schilddrüsendiagnostik selbst beigetragen $(3,8-16)$. Dies gilt vor allem auch für extrathyreoidale Miterkrankungen und Störungen der Proteinsynthese, die sich in Konzentrationsänderungen des TBG niederschlagen. Die Aussagekraft von Konzentrationsänderungen des TBG ist hoch, und über das Thyroxin/
Thyroxin bindende Globulin ( $\left.\mathrm{T}_{4} / \mathrm{TBG}\right)$-Verhältnis kann die Fraktion des freien $\mathrm{T}_{4}$ errechnet werden (14). Wir sehen in der getrennten Bestimmung von zwei kenngrößenabhängigen Größen einen großen klinischen Vorteil gegenüber der direkten Bestimmung von freiem $\mathrm{T}_{4}$. Die Problematik der TBGBestimmung liegt in ihrer bisher mangelnden Standạrdisierung. Aus Mangel an genügend hochreinem, chemisch gut charakterisierten TBG liegt keine einheitliche Referenzpräparation und damit kein gültiger Referenzbereich vor. Dies hat vor allem in der Klinik zu einer Unsicherheit gegenüber der TBG=Bestimmung geführt. Wir haben daher TBG erneut hochgereinigt und chemisch charakterisiert, worüber an anderer Stelle berichtet wird. Mit dem so gewonnenen TBG-Präparat wurde ein Enzymimmunoassay aufgebaut. In einer multizentrischen Studie wurden methodische und klinische Untersuchungen zur Êtablierung dieser TBG-Bestimmung durchgeführt, über die im folgenden berichtet werden soll!: 


\section{Material und Methoden}

Ionenaustauschermaterialien und Gelfiltrationsmedien wurden von der Firma Pharmacia, Freiburg, FRG, bezogen. Das Affinitätsadsorbens zur Immunosorption des anti-TBG wurde von Boehringer Mannheim (Best.-Nr. 665525) bezogen. Die Kopplung von TBG an den Adsorber (Spherosil) erfolgte nach mitgelieferter Vorschrift.

$L$-Thyroxin (freie Säure, p. A.) wurde von der Firma Henning, Berlin, FRG, bezogen. $L$-Thyroxin wurde an epoxyaktivierte Sepharose nach der von Kågedal \& Källberg (17) beschriebenen Methode gekoppelt.

Elektrophoresen wurden nach Lämmli (18) durchgeführt. Die Reduktion und Denaturierung des TBG erfolgte durch Inkubation mit Dithioerythrit $(10 \mathrm{~g} / \mathrm{l})$ und Erhitzen auf $100^{\circ} \mathrm{C}$ für 2 min. Die Aminosäure-Analyse des TBG erfolgte nach der Methode von Spackmann (19) an einem Beckman-AminosäureAnalysator $121 \mathrm{c}$. Die Mikroheterogenität des TBG wurde nach Gärtner (20) untersucht.

\section{TBG-Isolierung}

TBG wurde nach der Methode von Kågedal \& Källberg (17) mit einigen Modifikationen aus einem Pool von frischen Normalhumanseren isoliert.

Zunächst wurden Lipoproteine und Gerinnungsfaktoren durch Aerosilbehandlung aus dem Serum entfernt und die $\gamma$-Globulinfraktion durch Ammoniumsulfatfällung abgetrennt. Die weitere Aufreinigung erfolgte in 3 Schritten:

a) Immunsorption des TBG an $\mathrm{T}_{4}$-Epoxy-Sepharose und Elution mit 8-Anilinonaphthalin-sulfonsäure-(1) Ammoniumsalz

b) Diethylaminoethyl-Sephacel-Ionenaustausch-

Chromatographie

c) Sephacryl S 200-Chromatographie.

Die Ausbeute mit diesem Verfahren betrug 50\%. Die Reinheit des mit diesem Isolierverfahren erhaltenen TBG wurde in der SDS-Flachgel-Elektrophorese überprüft. Die Elektrophorese zeigte auch bei starker Überladung der TBG-Bande keine weiteren Banden. Unter den gewählten Bedingungen wäre eine Verunreinigung mit Fremdproteinen $>0,5 \%$ im Gel feststellbar gewesen.

\section{Charakterisierung des $\mathrm{TBG}$}

In der SDS-Gelelektrophorese zeigte das gereinigte TBG eine homogene Bande. Nach Behandlung mit Reduktionsmittel ergibt sich ebenfalls nur eine Bande mit leicht verringerter Wanderungsgeschwindigkeit im Vergleich zum nativen Molekül (21). Aus der SDS-Gelelektrophorese ergibt sich für das isolierte TBG ein Molekulargewicht von 59000 (22). Die Absorbanz einer TBG-Lösung $(10 \mathrm{~g} / \mathrm{l})$ bei $280 \mathrm{~nm}$ bei $1 \mathrm{~cm}$ Schichtdicke beträgt $7.0(2,5)$. Das gereinigte TBG bindet $T_{4}$ in einem molaren Verhältnis von 1:0,9 (23) und weist die in Tabelle 1 angegebene Aminosäurezusammensetzung auf.

In der isoelektrischen Fokussierung ergab das TBG im wesentlichen 3 Hauptbanden mit einem isoelektrischen Punkt von 4.18. 4.40 und 4,50 .

\section{Antiserumgewinnung}

Zur Gewinnung von TBG-Antiserum wurden Ziegen mit $10 \mu \mathrm{g}$ reinst-TBG in 1 ml komplettem Freund'schen-Adjuvans (Firma Difco) über 12 Monate einmal monatlich immunisiert. Anschließend wurde das Serum gesammelt und der Titer bestimmt. Die Titerbestimmung erfolgte durch die derjenigen Antiserumverdünnung. welche $30 \%$ des eingesetzten [ ${ }^{125}$ I] TBG in Gegenwart von Polyethylenglykol zu präzipitieren im Stande war.
Tab. 1. Aminosäureanalysen der eigenen TBG-Präparation in nmol bezogen auf 500 Aminosäurereste pro mol TBG. A) eigene Ërgebnisse

B) Ergebnisse $M . C$. Gershengorn et al. (2)

C) Ergebnisse K. Horn et al. (3).

\begin{tabular}{lrrr}
\hline & A & B & C \\
\hline Lysin & 35 & 28 & 28 \\
Histidin & 16 & 11 & 12 \\
Arginin & 8 & 6 & 6 \\
Asparaginsäure & 48 & 36 & 36 \\
Threonin & 33 & 25 & 24 \\
Serin & 39 & 29 & 30 \\
Glutaminsäure & 57 & 42 & 44 \\
Prolin & 22 & 15 & 16 \\
Glycin & 32 & 19 & 19 \\
Alanin & 37 & 28 & 28 \\
Cystein & 4 & 5 & - \\
Valin & 31 & 27 & 23 \\
Methionin & 11 & 12 & 20 \\
Isoleucin & 27 & 18 & 19 \\
Leucin & 59 & 38 & 39 \\
Phenylalanin & 28 & 22 & 22 \\
Tryptophan & - & 4 & - \\
Tyrosin & - & 9 & 9 \\
\hline
\end{tabular}

Die Spezifität des Antiserums wurde in der 2-dimensionalen Immunelektrophorese überprüft. Es zeigte keine Bande mit Human-Präalbumin und Human-Albumin sowie nur eine Bande mit Human-Schwangeren-Serum und normalem $\mathrm{Hu}-$ manserum. Eine unspezifische Reaktion des Antiserums mit $\mathrm{T}_{3}(100 \mu \mathrm{g} / \mathrm{l})$ und $\mathrm{T}_{4}(1000 \mu \mathrm{g} / \mathrm{l})$ konnte bei Verwendung im Enzymun-Test ${ }^{\oplus}$ TBG nicht festgestellt werden.

Anti-TBG-IgG wurde aus dem Serum durch Affinitätschromatographie an TBG-Spherosil isoliert. $700 \mathrm{ml}$ Serum wurden über eine 200-ml-TBG-Affinitätssäule aufgereinigt und das gebundene anti-TBG-IgG wurde durch Propionsäure (1 mol/l) eluiert.

\section{Standardisierung}

Für die Standardisierung von Enzym-Test ${ }^{\varpi}$ TBG wurden folgende Verfahren gewählt:

a) Isolierung des nativen Antigens in reiner Form,

b) Kalibrierung über Einwaage

Mit dem isolierten reinst-TBG, das in einer wäßrigen Lösung vorlag, wurde wegen der Instabilität des TBG in diesem Milieu in zeitlich genau koordinierten Aktionen folgende Bestimmung durchgeführt: Lyophilisation eines Aliquots der TBG-Lösung, Proteinbestimmung mit der Biuret-Methode, Herstellung von Urstandards.

In unabhängigen Verdünnungsansätzen wurden aus reinstTBG und TBG-freiem Serum Urstandards hergestellt. Alle nachfolgenden Standardchargen wurden an diesem Urstandard kalibriert.

\section{Herstellung von TBG-freiem Serum}

TBG wurde aus normalem Humanserum durch Affinitätschromatographie mittels $T_{4}$-Epoxysepharose (17) entfernt.

\section{Testablauf von Enzymun-Test ${ }^{\circledR}$ TBG}

$20 \mu \mathrm{l}$ Serum oder Standard werden zusammen mit $1 \mathrm{ml}$ Konjugat (TBG, gekoppelt an Meerrettich-Peroxidase) in mit anti- 
TBG beschichteten Polystyrolröhrchen für 60 min bei Raumtemperatur inkubiert. Nach B/F-Trennung durch Aussaugen der Flüssigkeit und einem Waschschritt wird die wandgebundene Konjugatmenge durch Zugabe von 1 ' $\mathrm{ml}$ 2,2'-Azino-di-(3ethyl-benzthiazolin-sulfonsäure(6)-diammoniumsalz (ABTS ${ }^{\circledR}$ ) für $60 \mathrm{~min}$ bestimmt. Das gefärbte Produkt wird bei $405 \mathrm{~mm}$ gemessen. Der ELISA TBG ist ein kompetitiver Test, d.h. die gemessene Absorbanz ist der TBG-Konzentration im Serum umgekehrt proportional.

\section{Aufbau der Studie}

12 klinisch-chemischen Instituten wurden vom Hersteller Testpackungen zur Bestimmung von Thyroxin bindendem Globulin $(\mathrm{TBG})^{1}$, Thyroxin $\left(\mathrm{T}_{4}\right)^{2}$ und Thyroxinbindungskapazität (TBK) ${ }^{3}$ zur Verfügung gestellt.

In 6 Laboratorien wurden die Testbestimmungen überwiegend manuell unter Zuhilfenahme von Pipettierhilfen durchgeführt, 6 Laboratorien setzten den ELISA-Meßplatz von Eppendorf ${ }^{4}$ ein.

Neben einem selbst entwickelten TBG-Radioimmunoassay (3) wurden Kits der folgenden Herstellèr verwendet: RIA-gnost ${ }^{\circledR}$ TBG, Behring-Werke AG; (125)J-TBG Radioimmunoassay Test System, Immophase, Corning; TBG-RIAcid, Henning GmbH; TBG Radioimmunoassay Kit, CEA SORIN; GammaDab (125) J-TBG Clinical Assay, Travenol.

Die Laboratorien werden in dieser Arbeit mit den Nummern $1-12$ bezeichnet. Die Numerierung ist nicht identisch mit der alphabetischen Reihenfolge der Teilnehmer.

Der $\mathrm{T}_{4} /$ TBG-Quotient wurde nach Glinoer $(8,14)$ als Quotient beider Konzentrationen, jeweils in $\mathrm{mg} / \mathrm{l}$, berechnet.

1) Im Handel erhältlich: Enzymun-Test ${ }^{\oplus}$ TBG, Art.-Nr. 249432, Firma Boehringer Mannheim GmbH.

$\left.{ }^{2}\right)$ Enzymun-Test ${ }^{\text {ig }}$ T4, Art.-Nr. 204510, Firma Boehringer Mannheim GmbH.

3) Enzymun-Test ${ }^{\oplus}$ TBK, Art.-Nr. 249416, Firma Boehringer Mannheim GmbH.

$\left.{ }^{4}\right)$ Eppendorf Gerätebau, Netheler \& Hinz GmbH, Hamburg.

\section{Ergebnisse}

\section{Präzision}

Die Präzision in der Serie (Abb.1), bestimmt mit nativem Humanserum im Konzentrationsbereich von 4-40 mg/l TBG und berechnet aus Mittelwerten von 3 fach Analysen, war zufriedenstellend (Variationskoeffizienten: $1,4-8,4 \%$ ):

Bei der Bestimmung der Präzision von Tag zu Tag mit einer aus Humanserum hergestellten Kontrollprobe ergaben sich Variationskoeffizienten von $1,5-8,9 \%$ bei einem Mittelwert von $13,0 \mathrm{mg} / 1$ TBG.

\section{Linearität}

Um zu überprüfen, ob eine lineare Beziehung zwischen Meßsignal und TBG-Konzentration besteht, wurden in unabhängigen Meß̈reihen 3 Humanseren $(36,1 ; 27,0 ; 21,2 \mathrm{mg} / 1 \mathrm{TBG}) \mathrm{mit}$ dem Inkubationspuffer (Phosphatpuffer $40 \mathrm{mmol} / \mathrm{l}, \mathrm{pH} 6,8,2,5 \mathrm{~g} / \mathrm{l}$ Rinderserumalbumin), dem TBG-freien Nullstandard der Testpackung sộwie mit $0,15 \mathrm{~mol} / 1 \mathrm{NaCl}$-Lösung stufenweise verdünnt.

Bei der Auftragung der gemessenen gegen die berech= neten TBG-Werte (Abb. 2) ergibt sich, daß zur Verdünnung TBG-freies Serum und $0,15 \mathrm{~mol} / 1 \mathrm{NaCl}-$ Lösung gleichermaßen eingesetzt werden können. Die mit Phosphatpuffer angesetżte Verdünnungsreihe zeigt einen sigmoiden Verlauf. Dieses Ergebnis konnte in 3 weiteren Laboratorien bestätigt werden.

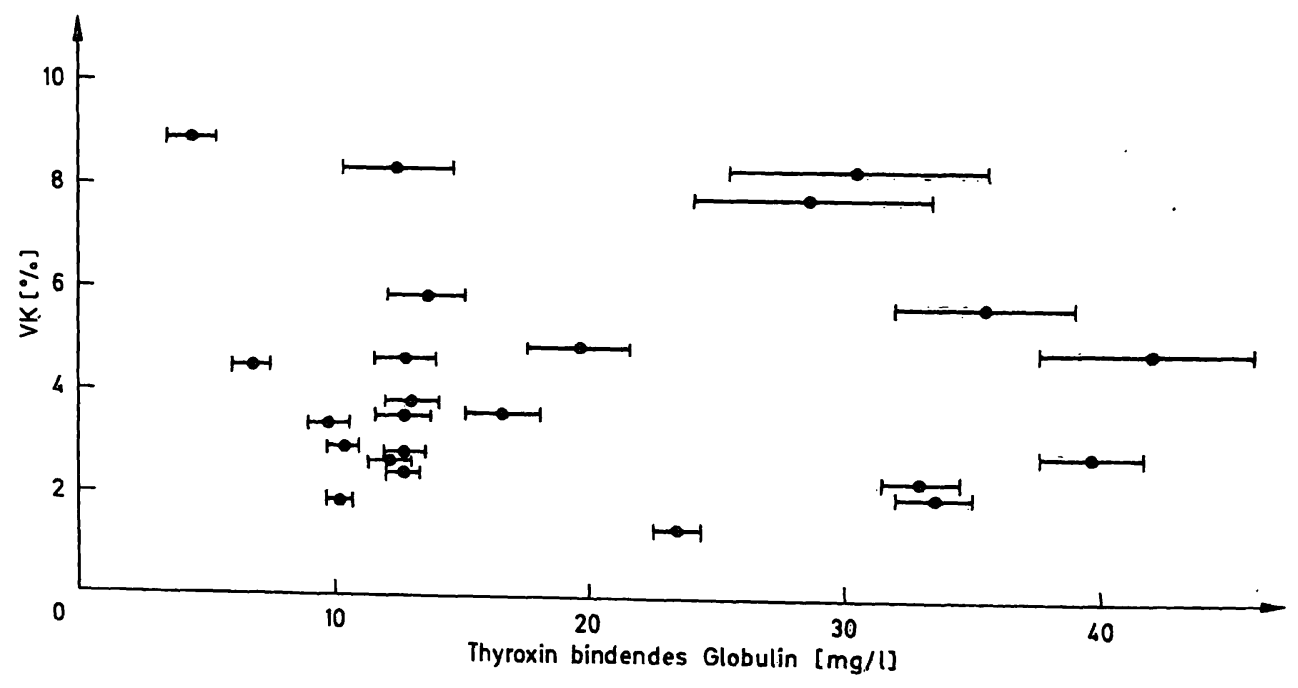

Abb. 1. Präzision in der Serie bei TBG-Konzentrationen von $4,4-40 \mathrm{mg} / 1$ (Zusammenfassung aller .Ẽrgebnisse). Es sind die Mittelwerte aus Dreifachbestimmungen $(\overline{\mathrm{x}} \pm 2 \mathrm{~s})$ dargestellt $(\mathrm{n}=10)$. 


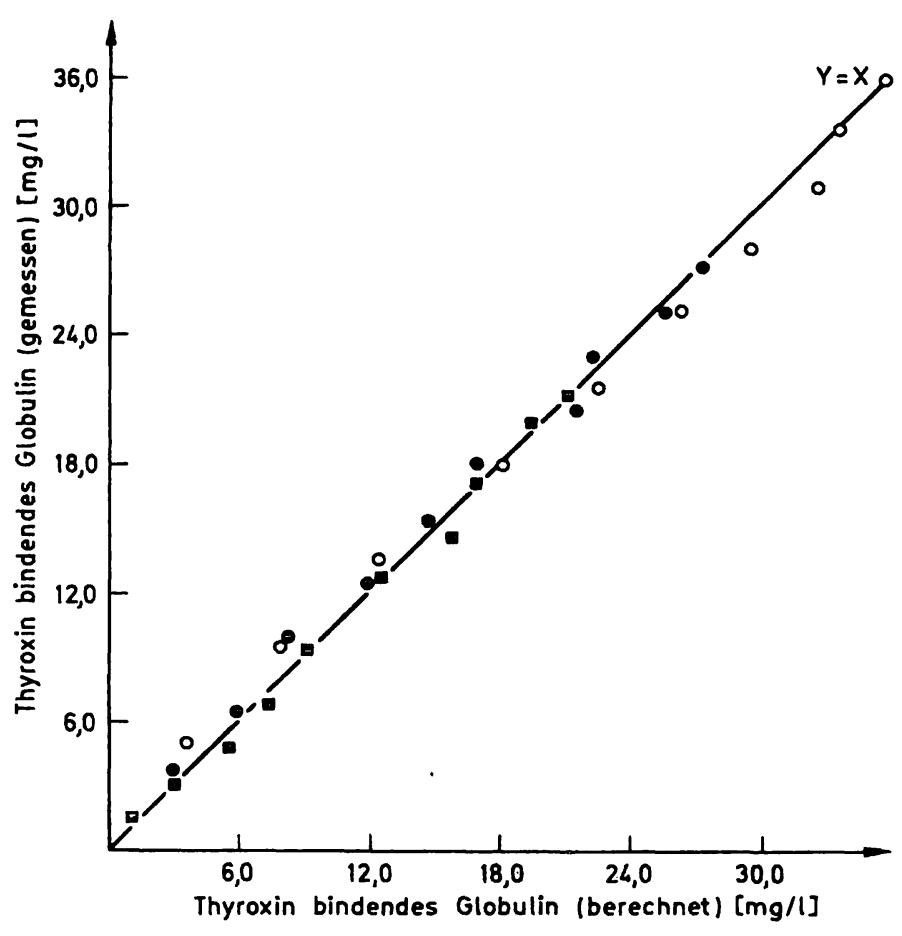

Abb. 2. Überprüfung der Linearität durch Verdünnung nativer Humanseren mit den Verdünnungsmedien:

O-O Phosphatpuffer, 国- TBG-freies Serum, O-O $0,15 \mathrm{~mol} / 1 \mathrm{NaCl}$-Lösung. Mittelwerte aus Dreifachanalysen.

\section{Methodenvergleich}

Der TBG-Enzymimmunoassay wurde von 1 Teilnehmer mit einem selbstentwickelten TBG-Radioimmunoassay (3) verglichen, 7 Laboratorien setzten kommerziell erhältliche TBG-Radioimmunoassays ein.

Die Ergebnisse wurden über ein nichtparametrisches Verfahren $(24,25)$ ausgewertet. Alle Vergleiche in Tabelle 2 zeigen deutlich, daß die Ergebnisse des Enzymimmunoassays systematisch um $20-40 \%$ niedriger liegen als die der Radioimmunoassays.

\section{Störsubstanzen}

\section{Arzneimittel}

Normalseren wurden mit der maximalen Tagesdosis (Herstellerangaben) von 37 häufig verwendeten Pharmaka (26) sowie Thiamazol und Dextrothyroxin versetzt. Eine Beeinflussung der Meßwerte im EnzymunTest ${ }^{\oplus}$ TBG konnte nicht festgestellt werden.

\section{Hyperbilirubinämie, Hyperlipämie}

Bei den mit dem Enzymimmunoassay und dem RIA der Firma Hennig gemessenen 35 Patientenseren mit Bilirubinkonzentrationen von $65-350 \mu \mathrm{mol} / 1$ und Triglyceridkonzentrationen von 3,5-17 mmol/l ergaben sich bei Berücksichtigung der methodischen Differenzen (Tab. 2) keine relevanten Unterschiede.

\section{Hämolyse}

Wie in Abbildung 3 dargestellt, wird der TBG-Enzymimmunoassay durch Hämoglobin gestört. Bei 10 $\mathrm{g} / \mathrm{l} \mathrm{Hb}$ werden etwa $2 \mathrm{mg} / \mathrm{l} \mathrm{TBG}$ weniger gemessen. Zusatzversuche zeigten, daß durch zweifaches Waschen in diesem Experiment nur noch $0.5 \mathrm{mg} / 1 \mathrm{TBG}$ zu wenig erfaßt werden. Eine mögliche Erklärung hierfür könnte eine unspezifische Bindung der aus den Erythrocyten freiwerdenden Pseudoperoxidase an den Antikörper sein, die eine zusätzliche Farbentwicklung verursacht und somit falsch $\mathrm{zu}$ niedrige TGB-Werte vortäuscht.

\section{Antikoagulantien}

Bei Überprüfung der Verwendbarkeit von Plasma (27) (Natriumfluorid, Natriumcitrat, EDTA, Heparin und Natriumoxalat) konnten im Vergleich zu den entsprechenden Seren in keinem Versuch Meßwertabweichungen gefunden werden.

Tab. 2. Methodenvergleiche von Enzymun-Test ${ }^{\oplus}$ TBG (y) mit verschiedenen RIA Kits (x) der angeführten Herstellerfirmen unter Anwendung eines nichtparametrischen Verfahrens.

\begin{tabular}{|c|c|c|c|c|c|c|c|}
\hline \multirow[t]{2}{*}{ Labor } & \multicolumn{2}{|l|}{ Methode } & \multirow[t]{2}{*}{$\left.\mathrm{N}^{1}\right)$} & \multirow{2}{*}{$\begin{array}{l}\text { Steigung } \\
\text { b }\end{array}$} & \multirow{2}{*}{$\begin{array}{l}\text { Achsen- } \\
\text { schnitt } \\
\text { a }\end{array}$} & \multirow{2}{*}{$\begin{array}{l}\text { TBG } \\
\overline{\mathrm{x}} \pm \mathrm{s} \\
(\mathrm{mg} / \mathrm{l})\end{array}$} & \multirow{2}{*}{$\begin{array}{l}\text { TGB } \\
\bar{y} \pm \mathbf{s} \\
(\mathrm{mg} / \mathrm{l})\end{array}$} \\
\hline & $\mathbf{x}$ & $y$ & & & & & \\
\hline 3 & Hyland & Enzymun-Test ${ }^{\infty}$ & 56 & 1,565 & 4,895 & $26,99 \pm 7,38$ & $17,07 \pm 4,75$ \\
\hline 4 & Henning & Enzymun-Test & 59 & 1,572 & 3,312 & $24,33 \pm 7,83$ & $16,11 \pm 4,99$ \\
\hline 5 & Henning & Enzymun-Test & 67 & 2,077 & 0,883 & $29,10 \pm 5,28$ & $16,93 \pm 3,56$ \\
\hline 6 & Behring & Enzymun-Test & 147 & 1,408 & 4,913 & $25,44 \pm 11,48$ & $17,04 \pm 7,57$ \\
\hline 8 & Henning & Enzymun-Test & 130 & 1,407 & 4,887 & $24,69 \pm 11,10$ & $16,88 \pm 8,15$ \\
\hline 10 & eig. RIA & Enzymun-Test & 53 & 1,979 & 2,825 & $29,61 \pm 8,94$ & $16,15 \pm 4,91$ \\
\hline 11 & Henning & Enzymun-Test ${ }^{\infty}$ & 52 & 1,488 & $-0,672$ & $26,19 \pm 13,97$ & $20,70 \pm 9,11$ \\
\hline 14 & Corning & Enzymun-Test ${ }^{\circledR}$ & 60 & 1,315 & 2,217 & $28,58 \pm 12,25$ & $20,31 \pm 9,31$ \\
\hline 14 & IDW & Enzymun-Test & 60 & 1,234 & $-0,037$ & $24,97 \pm 11,40$ & $20,31 \pm 9,31$ \\
\hline
\end{tabular}

\footnotetext{
1) Anzahl der Meßwertpaare
} 


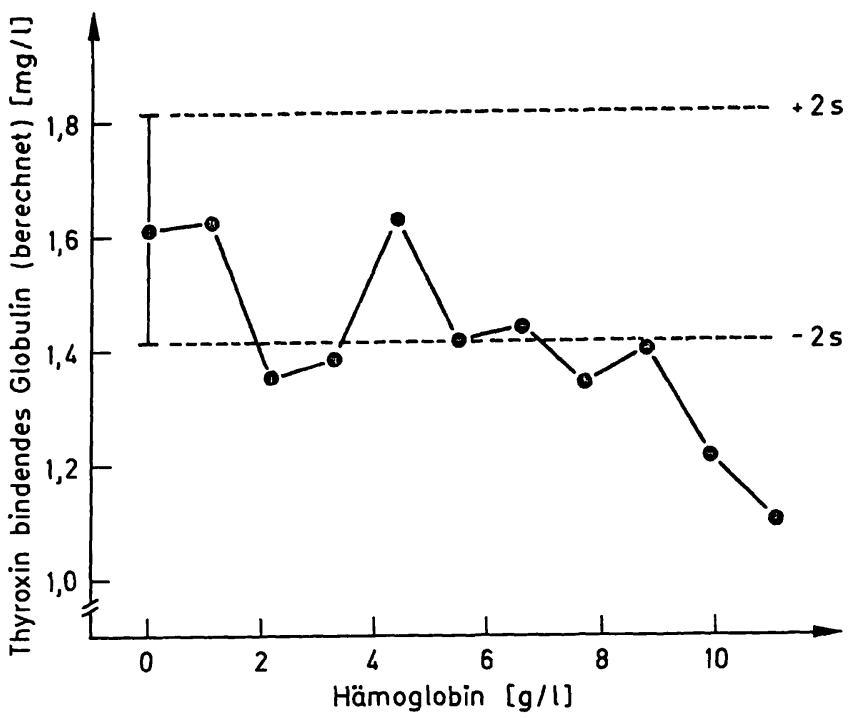

Abb. 3. Störung durch Hämoglobin. Bestimmung von TBG in einer Verdünnungsreihe aus hämolysefreiem und hämolytischem Serum des gleichen Patienten.
Referenzwerte für $T B G$ und $T_{4} / T B G-Q u o-$ tienten

Alle Teilnehmer unterteilten ihr Krankengut nach klinischen Gesichtspunkten, wobei die endgültige Diagnose aus der Gesamtheit zusätzlicher Kenngrößen erstellt wurde. Hierzu diente die Bestimmung von $T_{4}$, $\mathrm{T}_{3}, \mathrm{~T}_{3}$-uptake Test und die Bestimmung von Thyrotropin vor und nach Injektion von Protirelin (synthetisches Thyroliberin). Die Klassifikation erfolgte nach jeweils Labor-eigenen Referenzwertbereichen. Die Kollektive wurden eingeteilt in schilddrüsengesunde Kinder, Kontrollpersonen von 18-50 Jahren, Kontrollpersonen in einem Alter über 50 Jahren, Kranke mit blander Struma, hypo- und hyperthyreote Patienten, Frauen unter Östrogentherapie und Frauen in der Schwangerschaft. Bei schilddrüsengesunden Kontrollpersonen (18-50 Jahren) liegt der Median bei $14,3 \mathrm{mg} / 1 \mathrm{TBG}, 95 \%$ der Werte liegen zwischen

Tab. 3. Referenzwerte für TBG, ermittelt mit Enzym-Test ${ }^{\oplus}$ TBG in den Laboratorien aller Teilnehmer.

\begin{tabular}{|c|c|c|c|c|c|c|c|c|c|}
\hline & \multirow[t]{2}{*}{$\mathrm{N}$} & \multicolumn{8}{|c|}{ Thyroxin bindendes Globulin (mg/l) } \\
\hline & & Min & $5 \%$ & $10 \%$ & $50 \%$ & $90 \%$ & $95 \%$ & $\operatorname{Max}$ & $\overline{\mathbf{x}}$ \\
\hline \multicolumn{10}{|l|}{ Kinder } \\
\hline $\begin{array}{r}0-1 \text { Jahre } \\
1-7 \text { Jahre } \\
8-12 \text { Jahre } \\
13-17 \text { Jahre }\end{array}$ & $\begin{array}{l}17 \\
35 \\
20 \\
41\end{array}$ & $\begin{array}{r}9,3 \\
10,0 \\
12,1 \\
10,0\end{array}$ & $\begin{array}{r}9,3 \\
11,7 \\
12,6 \\
10,6\end{array}$ & $\begin{array}{l}10,5 \\
13,6 \\
13,0 \\
12,5\end{array}$ & $\begin{array}{l}19,3 \\
17,9 \\
16,8 \\
15,7\end{array}$ & $\begin{array}{l}30,7 \\
22,6 \\
20,0 \\
19,2\end{array}$ & $\begin{array}{l}31,2 \\
24,4 \\
20,9 \\
21,0\end{array}$ & $\begin{array}{l}21,2 \\
25,8 \\
21,0 \\
21,7\end{array}$ & $\begin{array}{l}19,4 \\
17,7 \\
16,8 \\
15,4\end{array}$ \\
\hline \multicolumn{10}{|c|}{ Schilddrüsengesunde erivachsene Kontrollpersonen } \\
\hline $\begin{array}{l}\text { gesamt } 18-50 \text { Jahre } \\
\text { davon Männer } \\
\text { davon Frauen }\end{array}$ & $\begin{array}{l}374 \\
196 \\
178\end{array}$ & $\begin{array}{l}6,6 \\
6,6 \\
9,4\end{array}$ & $\begin{array}{r}9,6 \\
9,2 \\
10,4\end{array}$ & $\begin{array}{l}10,5 \\
10,3 \\
10,9\end{array}$ & $\begin{array}{l}14,3 \\
13,4 \\
14,5\end{array}$ & $\begin{array}{l}17,8 \\
17,1 \\
17,9\end{array}$ & $\begin{array}{l}18,5 \\
17,8 \\
19,0\end{array}$ & $\begin{array}{l}21,5 \\
20,3 \\
21,5\end{array}$ & $\begin{array}{l}13,9 \\
13,4 \\
14,5\end{array}$ \\
\hline $\begin{array}{l}\text { gesamt }>50 \text { Jahre } \\
\text { davon Männer } \\
\text { davon Frauen }\end{array}$ & $\begin{array}{r}171 \\
72 \\
99\end{array}$ & $\begin{array}{l}9,2 \\
9,2 \\
9,8\end{array}$ & $\begin{array}{r}10,4 \\
9,5 \\
11,1\end{array}$ & $\begin{array}{l}11,1 \\
10,6 \\
12,1\end{array}$ & $\begin{array}{l}15,0 \\
14,2 \\
15,7\end{array}$ & $\begin{array}{l}18,4 \\
17,4 \\
18,6\end{array}$ & $\begin{array}{l}18,8 \\
18,3 \\
19,5\end{array}$ & $\begin{array}{l}23,1 \\
18,7 \\
23,1\end{array}$ & $\begin{array}{l}14,0 \\
14,4 \\
15,6\end{array}$ \\
\hline \multicolumn{10}{|l|}{ Hyperthyreose } \\
\hline $\begin{array}{l}\text { gesamt } \\
\text { davon Männer } \\
\text { davon Frauen }\end{array}$ & $\begin{array}{l}75 \\
18 \\
57\end{array}$ & $\begin{array}{l}7,9 \\
7,9 \\
8,0\end{array}$ & $\begin{array}{l}8,5 \\
7,9 \\
8,8\end{array}$ & $\begin{array}{r}10,5 \\
8,6 \\
10,5\end{array}$ & $\begin{array}{l}14,9 \\
13,1 \\
15,7\end{array}$ & $\begin{array}{l}18,5 \\
16,8 \\
18,7\end{array}$ & $\begin{array}{l}20,9 \\
20,9 \\
21,2\end{array}$ & $\begin{array}{l}26,5 \\
20,9 \\
26,5\end{array}$ & $\begin{array}{l}14,6 \\
13,4 \\
14,9\end{array}$ \\
\hline \multicolumn{10}{|l|}{ Hypothyreose } \\
\hline $\begin{array}{l}\text { gesamt } \\
\text { davon Männer } \\
\text { davon Frauen }\end{array}$ & $\begin{array}{l}74 \\
27 \\
47\end{array}$ & $\begin{array}{l}10,3 \\
10,6 \\
10,3\end{array}$ & $\begin{array}{l}11,8 \\
14,2 \\
11,8\end{array}$ & $\begin{array}{l}14,5 \\
14,6 \\
13,8\end{array}$ & $\begin{array}{l}19,5 \\
19,7 \\
19,5\end{array}$ & $\begin{array}{l}26,8 \\
24,5 \\
27,4\end{array}$ & $\begin{array}{l}29,4 \\
26,8 \\
29,5\end{array}$ & $\begin{array}{l}37,3 \\
28,8 \\
37,3\end{array}$ & $\begin{array}{l}20,0 \\
19,9 \\
20,0\end{array}$ \\
\hline \multicolumn{10}{|l|}{ Orale-Kontrazeptiva } \\
\hline & 83 & 14,2 & 16,4 & 17,8 & 22,2 & 29,1 & 30,6 & 32,2 & 23,4 \\
\hline \multicolumn{10}{|l|}{ Schwangerschaft } \\
\hline $\begin{array}{l}\text { gesamt } \\
\text { davon bis } 26 . \text { Woche } \\
\text { davon } 27 .-40 . \text { Woche }\end{array}$ & $\begin{array}{l}46 \\
12 \\
34\end{array}$ & $\begin{array}{l}17,4 \\
23,4 \\
22,3\end{array}$ & $\begin{array}{l}22,7 \\
23,4 \\
22,7\end{array}$ & $\begin{array}{l}23,8 \\
23,7 \\
25,2\end{array}$ & $\begin{array}{l}28,5 \\
24,5 \\
29,5\end{array}$ & $\begin{array}{l}36,9 \\
35,0 \\
36,9\end{array}$ & $\begin{array}{l}37,7 \\
37,8 \\
40,2\end{array}$ & $\begin{array}{l}40,9 \\
37,8 \\
40,9\end{array}$ & $\begin{array}{l}29,2 \\
28,3 \\
29,9\end{array}$ \\
\hline Dialyse & & & & & & & & & $\cdot$ \\
\hline gesamt & 33 & 6,9 & 10,8 & 11,4 & 15,5 & 20,8 & $30 ; 7$ & 32,5 & 16,3 \\
\hline
\end{tabular}




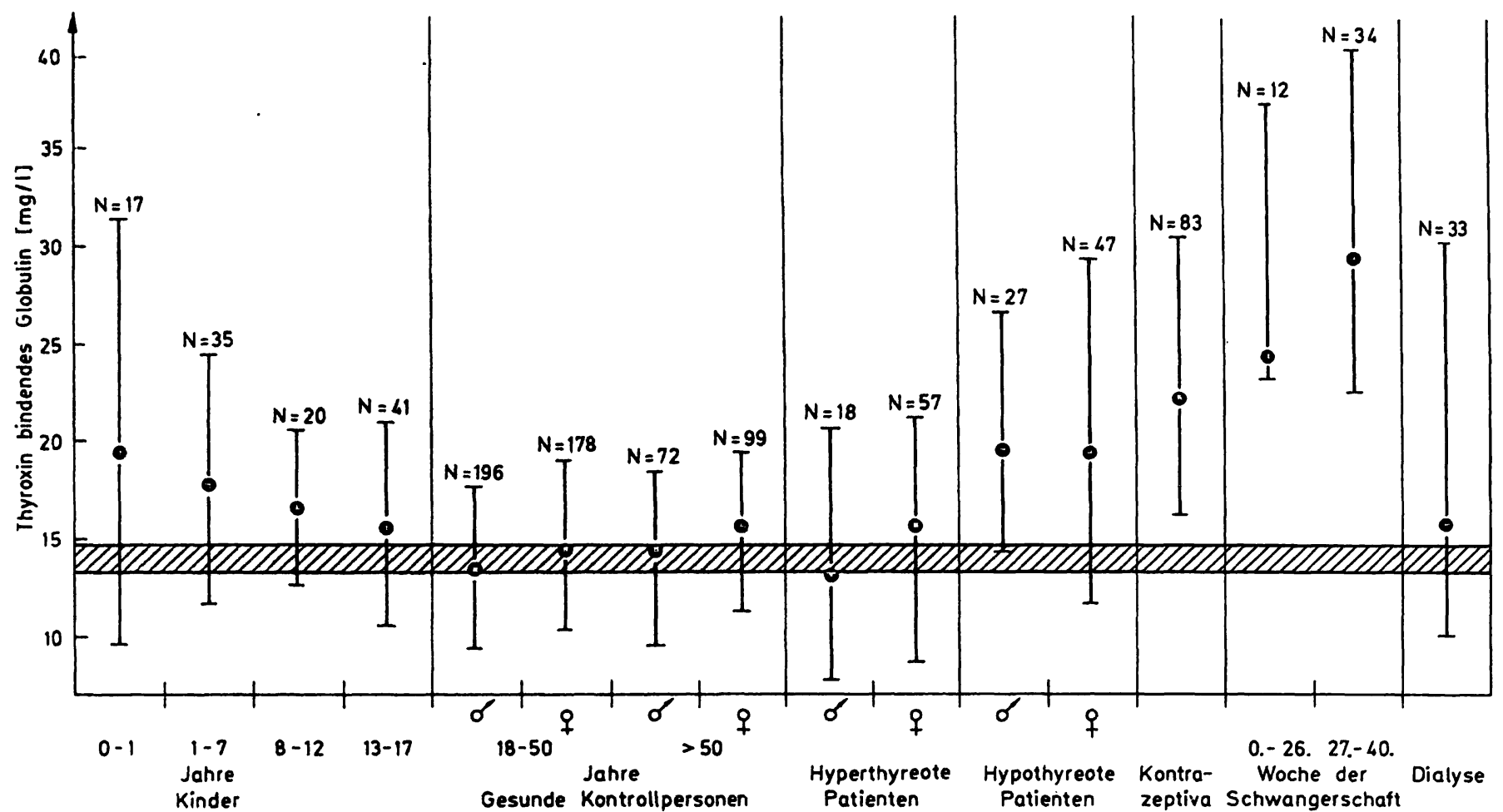

Abb. 4. Referenzwerte für TBG. Es sind die Medianwerte (O) und die $95 \%$ Bereiche (I) der einzelnen Kollektive aufgetragen. Der Referenzbereich schilddrüsengesunder Probanden einer mittleren Altersgruppe ist schraffiert eingezeichnet.

9,6-18,5 mg/1 TBG. Der Median bei Frauen mit 14,5 mg/l TBG lag signifikant höher als bei Männern mit $13,4 \mathrm{mg} / \mathrm{l}$ TBG. Die Werte hyperthyreoter Patienten liegen überwiegend im Referenzbereich, hypothyreote Patienten zeigen bei einem Median von $19,5 \mathrm{mg} / \mathrm{l}$ TBG deutlich erhöhte Werte (Tab. 3, Abb. 4).

Durch die Bildung von $\mathrm{T}_{4} / \mathrm{TBG}$-Quotienten (Tab. 4 , Abb. 5) erhält man bei gesunden Kontrollpersonen (18 - 50 Jahren) einen Medianwert von 4,6, bei hypothyreoten Patienten von 1,0 und bei hyperthyreoten Patienten von 11,3. In der Schwangerschaft liegen die Quotienten (Median = 3,1) an der oberen Grenze des hypothyreoten Bereichs.

\section{Diskussion}

Die sinnvolle Kombination von Laboruntersuchungen zur rationalen und richtigen Diagnostik von Erkrankungen der Schilddrüse und deren metabolischen peripheren Konsequenzen mit hoher Sensitivität und Spezifität ist immer noch kontrovers. Die Bedeutung der Bestimmung der Gesamt-SchilddrüsenhormonKonzentrationen ist unbestritten $(3,8-16)$. Ungewiß ist, ob zur Miterfassung der freien Hormonfraktion deren direkte Bestimmung nützlich ist $(28,29)$ oder ob über eine quantitative Erfassung der Konzentration der wesentlichen Bindungsproteine die Errechnung der freien Hormonfraktion vorzuziehen ist (3,
$8,11,15)$. In dieser Situation erscheint es zunächst bedeutsam, über eine reproduzierbare Methodik zur quantitativen Bestimmung des wichtigsten Schilddrüsenhormon-Bindungsproteins, des Thyroxin bindenden Globulins (TBG) zu verfügen. Ein wesentliches Problem bei der Quantifizierung von TBG ist das Fehlen eines internationalen Referenzpräparates oder eines allgemein akzeptierten hochgereinigten TBG-Standard-Präparates. Wir haben das Glykoprotein TBG noch einmal hochgereinigt und chemisch charakterisiert. Die unterschiedlichen chromatographischen Techniken der verschiedenen Arbeitsgruppen $(2,3,5)$ führen offenbar zu unterschiedlich zusammengesetzten Proteinen, was sich an der unterschiedlichen Aminosäurezusammensetzung ablesen läßt. Aus früheren präparativen Untersuchungen war schon bekannt, daß ein unterschiedliches Molekulargewicht von etwa $60000 \pm 3000$ angegeben wurde $(1,4,20,21,22)$. Obwohl angenommen wurde, daß TBG aus 374 Aminosäuren zusammengesetzt ist, und zur einen Hälfte aus einer $\alpha$-Helix und zur anderen Hälfte aus einer $\beta$-Faltblatt-Struktur bestehen sollte, besteht aufgrund der präparativen Unsicherheit und der Mikroheterogenität von $\operatorname{TBG}(4,22)$ noch immer eine Unsicherheit hinsichtlich der molekularen Zusammensetzung. Dies ist bedeutend, da insbesondere die Beteiligung hydrophiler Proteinsequenzen an einem Protein dessen antigene Determination bestimmen (30). Obwohl bei unserer Präpara- 
Tab. 4. Referenzwerte für den T4/TBG-Quotienten.

\begin{tabular}{|c|c|c|c|c|c|c|c|c|c|}
\hline & \multirow[t]{2}{*}{$\mathrm{N}$} & \multicolumn{8}{|c|}{ T4/TBG-Quotienten } \\
\hline & & Min & $5 \%$ & $10 \%$ & $50 \%$ & $90 \%$ & $95 \%$ & $\operatorname{Max}$ & $\overline{\mathbf{x}}$ \\
\hline \multicolumn{10}{|c|}{ Schilddrüsengesunde erv'achsene Kontrollpersonen } \\
\hline $\begin{array}{l}\text { gesamt } 18-50 \text { Jahre } \\
\text { davon Männer } \\
\text { davon Frauen }\end{array}$ & $\begin{array}{l}323 \\
181 \\
142\end{array}$ & $\begin{array}{l}2,9 \\
2,9 \\
2,9\end{array}$ & $\begin{array}{l}3,4 \\
3,6 \\
3,4\end{array}$ & $\begin{array}{l}3,6 \\
3,8 \\
3,5\end{array}$ & $\begin{array}{l}4,6 \\
4,8 \\
4,4\end{array}$ & $\begin{array}{l}6,2 \\
6,2 \\
6,0\end{array}$ & $\begin{array}{l}6,5 \\
6,5 \\
6,4\end{array}$ & $\begin{array}{l}14,6 \\
10,2 \\
14,6\end{array}$ & $\begin{array}{l}4,8 \\
4,9 \\
4,6\end{array}$ \\
\hline $\begin{array}{l}\text { gesamt }>50 \text { Jahre } \\
\text { davon Männer } \\
\text { davon Frauen }\end{array}$ & $\begin{array}{r}156 \\
66 \\
90\end{array}$ & $\begin{array}{l}2,8 \\
3,1 \\
2,8\end{array}$ & $\begin{array}{l}3,3 \\
3,7 \\
3,2\end{array}$ & $\begin{array}{l}3,7 \\
3,8 \\
3,6\end{array}$ & $\begin{array}{l}4,9 \\
4,9 \\
4,9\end{array}$ & $\begin{array}{l}6,2 \\
6,3 \\
6,1\end{array}$ & $\begin{array}{l}6,6 \\
6,5 \\
6,6\end{array}$ & $\begin{array}{r}12,9 \\
7,3 \\
12,9\end{array}$ & $\begin{array}{l}4,9 \\
4,9 \\
4,9\end{array}$ \\
\hline \multicolumn{10}{|l|}{ Hyperthyreose } \\
\hline $\begin{array}{l}\text { gesamt } \\
\text { davon Männer } \\
\text { davon Frauen }\end{array}$ & $\begin{array}{l}62 \\
15 \\
47\end{array}$ & $\begin{array}{l}4,0 \\
7,9 \\
4,0\end{array}$ & $\begin{array}{l}7,7 \\
7,9 \\
5,9\end{array}$ & $\begin{array}{l}8,0 \\
8,8 \\
7,8\end{array}$ & $\begin{array}{l}11,3 \\
11,3 \\
11,1\end{array}$ & $\begin{array}{l}20,7 \\
17,5 \\
20,9\end{array}$ & $\begin{array}{l}23,5 \\
22,0 \\
24,3\end{array}$ & $\begin{array}{l}30,3 \\
22,0 \\
30,3\end{array}$ & $\begin{array}{l}12,7 \\
12,3 \\
12,8\end{array}$ \\
\hline \multicolumn{10}{|l|}{ Hypothyreose } \\
\hline $\begin{array}{l}\text { gesamt } \\
\text { davon Männer } \\
\text { davon Frauen }\end{array}$ & $\begin{array}{l}70 \\
24 \\
46\end{array}$ & $\begin{array}{l}0,1 \\
0,1 \\
0,1\end{array}$ & $\begin{array}{l}0,2 \\
0,2 \\
0,5\end{array}$ & $\begin{array}{l}0,4 \\
0,2 \\
0,6\end{array}$ & $\begin{array}{l}1,0 \\
0,8 \\
1,1\end{array}$ & $\begin{array}{l}3,7 \\
2,7 \\
3,7\end{array}$ & $\begin{array}{l}3,9 \\
3,9 \\
3,9\end{array}$ & $\begin{array}{l}4,6 \\
4,3 \\
4,6\end{array}$ & $\begin{array}{l}1,5 \\
1,1 \\
1,7\end{array}$ \\
\hline \multicolumn{10}{|l|}{ Orale Kontrazeptiva } \\
\hline & 49 & 2,2 & 2,7 & 2,8 & 3,7 & 4,9 & 5,0 & 5,5 & 3,8 \\
\hline \multicolumn{10}{|l|}{ Schw'angerschaft } \\
\hline $\begin{array}{l}\text { gesamt } \\
\text { davon bis } 26 . \text { Woche } \\
\text { davon } 27 .-40 \text {. Woche }\end{array}$ & $\begin{array}{l}46 \\
12 \\
34\end{array}$ & $\begin{array}{l}2,3 \\
2,6 \\
2,3\end{array}$ & $\begin{array}{l}2,4 \\
2,6 \\
2,4\end{array}$ & $\begin{array}{l}2,5 \\
2,8 \\
2,5\end{array}$ & $\begin{array}{l}3,1 \\
3,5 \\
3,1\end{array}$ & $\begin{array}{l}4,0 \\
4,6 \\
3,9\end{array}$ & $\begin{array}{l}4,5 \\
4,6 \\
4,0\end{array}$ & $\begin{array}{l}4,8 \\
4,6 \\
4,2\end{array}$ & $\begin{array}{l}3,3 \\
3,6 \\
3,6\end{array}$ \\
\hline \multicolumn{10}{|l|}{ Dialyse } \\
\hline gesamt & 33 & 2,1 & 2,1 & 2,4 & 3,5 & 5,4 & 6,8 & 7,4 & 3,8 \\
\hline
\end{tabular}

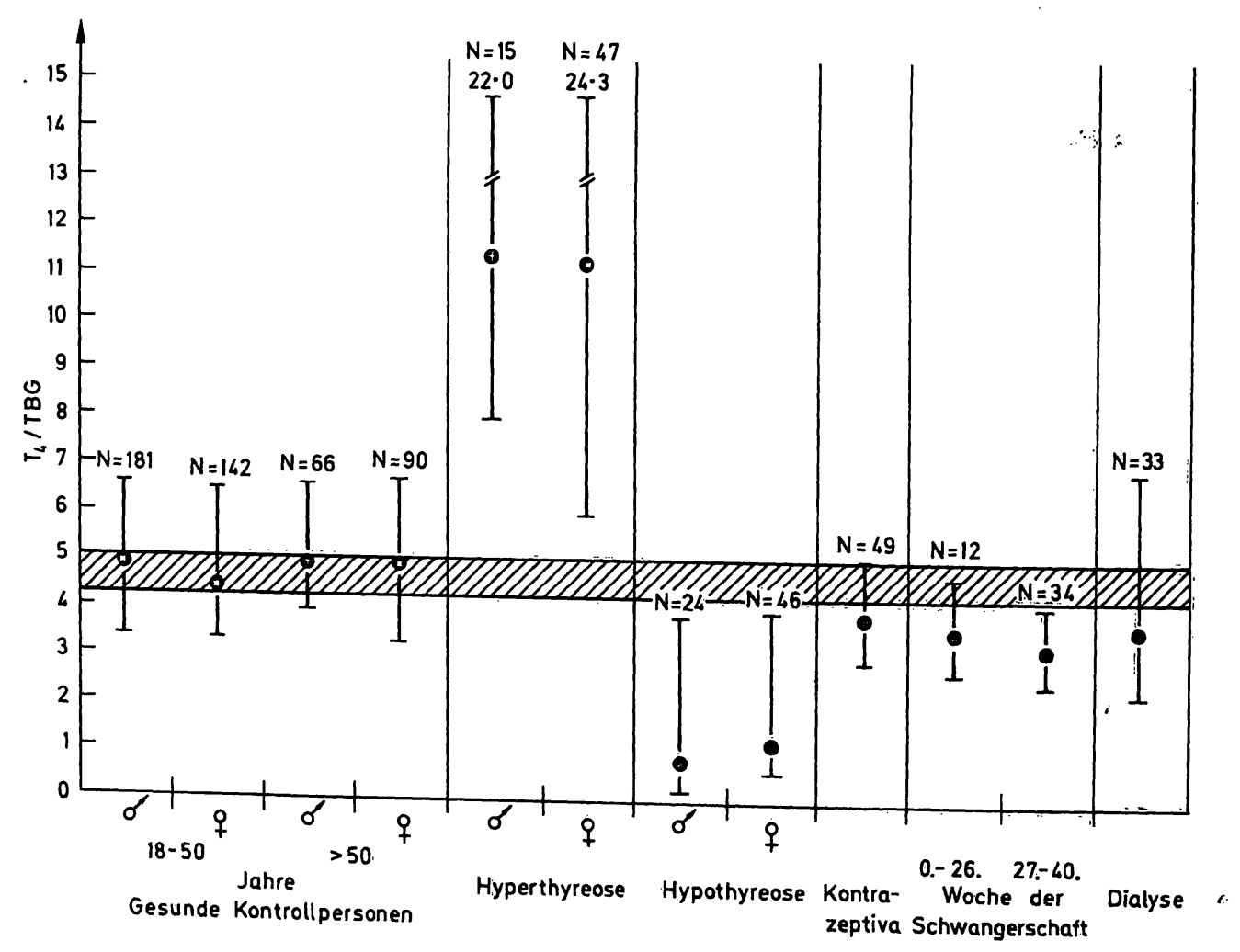

Abb. 5. Referenzwerte für $T_{4} /$ TBG-Quotienten. Es sind die Medianwerte (O) und die $95 \%$ Bereiche (I) der einzelnen Kollektive aufgetragen. Der Referenzbereich schilddrüsengesunder Probanden einer mittleren Altersgruppe ist schraffiert eingezeich-
net.

J. Clin. Chem. Clin. Biochem. / Vol. 23, 1985 / No. 3 
tion der heutige Standard der Proteinchemie berücksichtigt wurde, ist die Reproduzierbarkeit der Präparation nicht gewährleistet. Daher ist dieses TBG nicht als Referenzstandard verwendbar. Vielmehr empfiehlt sich zur Vereinheitlichung unterschiedlicher TBG-Bestimmungsmethoden die internationale Etablierung eines Poolserum-Standards mit Konzentrationsangabe.

Dieses Vorgehen wäre aber nur zu vertreten, wenn Antiseren, die mit unterschiedlichen TBG-Präparationen hergestellt wurden, bei paralleler Verdünnung identische Meßergebnisse mit einer solchen SerumPräparation zeigen würden.

Unser Methodenvergleich hat erwiesen, daß die Immunreaktion unterschiedlicher Antiseren gegen die antigenen Hauptdeterminanten von im Serum zirkulierendem TBG parallel ist, so daß dieses Vorgehen sinnvoll ist und damit erwartet werden kann, daß alle kommerziellen Testbestecke mit einem SerumReferenzstandard identische Werte produzieren. Damit ist eine wesentliche Voraussetzung für die klinische Praktikabilität und die Weiterverbreitung der TBG-Bestimmung gegeben.

Theoretische Berechnungen lassen die Konzentration von zirkulierendem TBG im Serum bei $10 \mathrm{mg} / 1$ erwarten $(1,21,23)$. Die von anderen Autoren $(9,10,31)$ radioimmunchemisch ermittelten Konzentrationen bei gesunden Probanden lagen nahe an diesem Erwartungswert, der auch mit unserer enzymimmunologischen Methode annäherungsweise gemessen wird. Von mehreren Autoren wurden aber teilweise wesentlich höhere Konzentrationen angegeben $(6,3,11,32)$. Dieser Unterschied wäre nicht gravierend, wenn nicht die TBG-Bestimmung zur Errechnung der freien T4Fraktion mittels des T4/TBG-Quotienten herangezogen würde $(8,14)$. Eine standardisierbare Vereinheitlichung ist daher erforderlich. Die hier vorgestellte enzymimmunologische Bestimmungsmethode weist eine hohe methodische Präzision auf und hat in der klinischen Praxis eine große Zuverlässigkeit gezeigt. Die klinische Brauchbarkeit läßt sich auch an der geringen Störanfälligkeit gegenüber relevanten Interferenzen ausweisen. Die Bestimmung der TBG-Konzentration bei Kontrollpersonen zeigt die bekannte Abhängigkeit vom Lebensalter $(12,33)$. Von der perinatalen Lebensphase an bis zum Erwachsenenalter nimmt die TBG-Konzentration zunächst kontinuierlich ab, um nach dem 50 . Lebensjahr wieder anzusteigen. Offensichtlich haben aber solche altersabhängigen TBG-Schwankungen keinen Einfluß auf die Bioverfügbarkeit von Schilddrüsenhormonen. Die häufigste Ursache für die Erhöhung der TBG-Konzentration ist die Östrogenwirkung auf Synthese in der Leber und peripheren Abbau von TBG $(3,20)$. So- wohl unter einer erhöhten endogenen Östrogen-Produktion während der Schwangerschaft als auch unter exogener Zufuhr von Östrogenen etwa in der Form von oralen Kontrazeptiva finden sich Anstiege der TBG-Konzentrationen, die sich nach Beendigung von Schwangerschaft oder Östrogen-Medikation wieder normalisieren $(8,34)$.

Der Enzymimmunoassay zeigt diese bekannten Veränderungen zuverlässig an. Der Verlauf von TBGKonzentrationsänderungen bei akuten und chronischen Lebererkrankungen ist gegenläufig. Bei akuter Hepatitis kann es zu einem Anstieg von TBG kommen, während mit zunehmender Verminderung der Lebersyntheseleistung die TBG-Konzentration abfällt $(11,35)$. Genetisch bedingte TBG-Vermehrungen sind beschrieben (36), ebenso erhöhte Konzentrationen von TBG bei Hypothyreose $(8,22)$. Unter dem Einfluß von Androgenen (35), hochdosierter SteroidMedikation (37), im Verlauf von schweren Allgemeinerkrankungen $(7,13)$, bei anhaltenden katabolen Stoffwechselzuständen (11), bei Null-Diät (38) sowie bei genetisch bedingtem TBG-Mangel $(11,39)$ und bei schwerer Hyperthyreose können verminderte TBGKonzentrationen gefunden werden.

Die TBG-Messung ergibt also eine zuverlässige Konzentrationsangabe des wichtigsten zirkulierenden Schilddrüsenhormon-Bindungsproteins, dessen Konzentrationsänderungen für die Diagnostik von Schilddrüsenerkrankungen und Änderungen des peripheren Schilddrüsenhormonstoffwechsels unter Einbeziehung der Gesamtkonzentrationen an Schilddrüsenhormonen relevant werden kann. Dies gilt besonders für T4, wobei eine Kenngröße für dessen freie Fraktion klinisch bedeutsam ist. Durch Bildung des T4/TBG-Quotienten $(8,14)$ kann frei von physikochemischen Voraussetzungen an die Definition des freien T4 eine Kenngröße für diese biologisch und klinisch relevante Meßgröße T4 ermittelt werden. Damit sind die immer noch kontroversen Diskussionen um die methodische Richtigkeit der direkten Bestimmung des freien T4 sowie ihre Beeinflussung durch Serumbestandteile oder durch andere Krankheiten umgangen, und die Qualität der Schilddrüsen-Diagnostik wird nicht durch die Unsicherheit eines Testsystems in Frage gestellt. Vielmehr erlaubt die getrennte Ermittlung von TBG und freiem T4 eine kausale Zuordnung klinisch relevanter Abweichungen vom Referenzbereich. Unsere Untersuchungen bestätigen, daß Änderungen von TBG die mit diesem Protein im Gleichgewicht stehende freie T4-Fraktion kaum beeinflußt. Das gilt insbesondere für altersabhängige Schwankungen. Bemerkenswert ist, daß der T4/TBG-Quotient in der Gravidität signifikant gegenüber dem Referenzbereich erniedrigt ist, so daß 
auch diese Untersuchung wieder die Frage aufwirft, ob während der Gravidität eine leichte Hypothyreose des mütterlichen Organismus besteht (40).

Bei nierenkranken Patienten kann es je nach Funktionszustand der Niere zu komplexen Änderungen der Bindungsproteine und der SchilddrüsenhormonKonzentrationen kommen, wobei der T4/TBG-Quotient eine hypothyreote Stoffwechsellage zuverlässig anzeigt (41). Obwohl die Einzeldaten aus unserer Untersuchung hier nicht angeführt sind, so unterstützt unsere Untersuchung das Ergebnis zahlreicher Untersucher, die fanden, daß die Treffsicherheit des T4/TBG-Quotienten bei der Abgrenzung grenzwertiger Situationen klinisch Hyper- oder Hypothyreosen als höher angesehen werden kann als die des FT4Index, des ETR- oder des NTR-Tests $(3,8,11,13,14$, $32,35)$. Nur einzelne Autoren halten die genannten Kenngrößen in der klinischen Routine-Diagnostik für gleichwertig (42), oder geben dem FT4-Index gegenüber dem T4/TBG-Quotienten den Vorzug (43). Die hohe Treffsicherheit von T4/TBG-Quotient unterliegt einigen Einschränkungen, insbesondere gehen Änderungen von Albumin und Präalbumin nicht in die Berechnungen ein. Dies ist meist ohne klinischen Belang, solange TBG das bestimmende Bindungsprotein mit der höchsten Affinität für T4 ist. In jüngster Zeit ist aber eine T4 Erhöhung durch eine spezifische T4 bindende Albumin-Fraktion beschrieben worden, deren diagnostische Relevanz für den Schilddrüsenhormonstoffwechsel von kritischer Bedeutung sein kann (44). Da die Bindung von T3 an TBG mit einer geringeren Affinität als die von T4 erfolgt, läßt sich eine Kenngröße für das freie T3 zwar errechnen, erreicht aber keine diagnostische Trennschärfe. Mit Rücksicht darauf, da $B$ T3 hauptsächlich die periphere Konversion von T4 anzeigt, ist aber aus pathophysiologischen Gründen ein freies T3, sofern es korrekt definiert werden könnte, klinisch auch nicht so relevant, wie der nicht an Protein gebundene Anteil von T4. Für den T4/TBG-Quotienten gelten somit folgende diagnostische Grenzen.

1. bei einem stark ausgeprägten TBG-Mangel steigt der T4/TBG-Quotient disproportioniert an, vermutlich weil das Thyroxin bindende Präalbumin stärker mit Thyroxin abgesättigt ist als bei normalem TBG-Gehalt (45).
2. der T4/TBG-Quotient gibt keinen direkten Aufschluß über eine mögliche Verdrängung von Schilddrüsenhormonen aus der Proteinbindung durch Medikamente (z. B. Salicylat, Phenytoin) oder Metabolite (freie Fettsäuren), sondern läßt diesen Verdacht bestenfalls durch einen unerklärlich niedrigen Wert aufkommen'. Diese Einschränkungen gelten aber auch für die anderen Bestimmungsmethoden von freiem $\mathrm{T} 4$.

\section{Schlußfolgerungen}

Die hier vorgestellte immunochemische Bestimmung von TBG (Enzymun-Test ${ }^{\circledR}$ TBG) ist eine empfindliche, zuverlässige und klinisch relevante Methode zur Messung der zirkulierenden TBG-Kónzentrationen im menschlichen Serum. Durch die Kalibrierung an einem gut charakterisierten Referenzpräparat konnte für Kinder und Erwachsene beiderlei Geschlechts ein Referenzbereich erarbeitet werden. Die= ser Referenzbereich für TBG und die weitgehende Standardisierung der Gesamt-Thyroxin-Bestimmung führen dann ebenfalls zu einem Bereich für den T4/ TBG-Quotienten, womit eine wesentliche Voraussetzung für eine Vereinheitlichung der klinischen Evaluierung gegeben ist. Der T4/TBG-Quotient erlaubt es, ein physiko-chemisch gut charakterisiertes Äquivalent für das freie T4 anzugeben, das verläßlicher erscheint als die unterschiedlichen direkten Bestimmungsmethoden für freies T4. Die Bestimmung von TBG und die Ermittlung von freiem T4 aus T4/TBG ist für die Erstdiagnostik thyreogener und extrathyreoidaler Störungen bei Kindern und Frauen bei unbekannter östrogener Ausgangslage unerläßlich. Die Anwendung erscheint ebenfalls sinnvoll bei allen Patienten, bei welchen neben der Schilddrüsenkrankheit a priori eine extrathyreoidale Erkrankung mit in die Differentialdiagnose einbezogen werden muß, da dann der Protirelin-Test in seiner Aussage eingeschränkt sein kann. TBG-Bestimmung und Protirelin-Test ergänzen sich also schon bei der Erstdiagnose einer Schilddrüsenerkrankung, während die TBG-Bestimmung allein zusammen mit der Bestimmung von Schilddrüsenhormonen für alle Verlaufsuntersuchungen zur Behandlung von Schilddrüsenerkrankungen und extrathyreoidalen Erkrankungen eine relevante Methode ist. 


\section{Literatur}

1. Sterling. K.. Hamada, S., Takemura, Y., Brenner, M. A., Newman, E.S. \& Inada, M. (1971), J. Clin. Invest. 50, $1758-1971$.

2. Gershengorn, M.C., Cheng, S. Y., Lippoldt, R. E., Lord, R.S. \& Robbins, J. (1977) J. Biol. Chem. 252, 8713-8718.

3. Horn. K.. Kubiczek, Th., Pickardt, C. R., Scriba, P.C. (1977) Klin. Wochenschr. 55, $881-894$.

4. Cheng. C.-Y. (1977) Biochem. Biophys. Res. Commun. 79, $1212-1218$.

5. Hocman. G. (1978) Int. J. Biochem. 9, 295-298.

6. Cavalieri. R. R. (1975) J. Clin. Invest. 56, 79-87.

7. Tabachink, M. \& Korcek, L. (1978) Biochim. Biophys. Acta 537. $169-175$.

8. Glinoer. D., Fernande-Deville, M. \& Ermans, A. M. (1978) J. Endocrinol. Invest. 1, 329-335.

9. Kågedal. B. \& Kaellberg, M. (1977) Clin. Chem. 23, 1694-1699.

10. Gershengorn. M. C., Larsen, P. R. \& Robbins, J. (1976) J. Clin. Endocrinol. Metab. 42, 907-911.

11. Pickardt. C. R.. Bauer, M., Horn, K., Kubiczek, Th. \& Scriba. P. C. (1977) Internist $18,538-543$.

12. Hesch. R. D.. Gatz, J., Jueppner, H. \& Stuppe, P. (1977) Horm. Metab. Res 9, 141-146.

13. McDowell. D. R. (1979) Ann. Clin. Biochem. 16, 81-85.

14. Hesch. R. D. (1977) Dtsch. Med. Wochenschr. 102, $1386-1388$.

15. Horn. K.. Pickard, C. R. \& Scriba, P.C. (1979) Nuclearmediziner 2, 17 .

16. Rudorff, K.-H., Hermann, J., Kroell, H. J. \& Krueskemper, H. L. (1976) J. Clin. Chem. Clin. Biochem. 14, 31-36.

17. Kågedal. B. \& Kaellberg, M. (1977) Clin. Chim. Acta 78, $103-111$.

18. Lämmli. M. K. (1970) Nature 227, 680-685.

19. Spackmann, M., Stein, D. H. \& Moore, W. H. (1958) Anal. Chem. 30. 1190-1206.

20. Gärtner. R., Henze, R., Horn, K., Pickardt, C. R. \& Scriba, P. C. (1981) J. Clin. Endocrinol. Metab. 52, 657-664.

21. Marshall, J.S. \& Pensky J. (1971) Arch. Biochem. Biophys. 146. 76-83.

22. Raouf, A. A. M., Geisow, M.J., O'Gorman, P., Marsden, P. \& Howorth, P. (1980) Clin. Chim. Acta 104, 25-41.

23. Green. A. M., Marshall, J.S., Pensky, J. \& Stanbury, J. (1972) Biochim. Biophys. Acta 278, 117-124.

24. Passing. H. \& Bablock, W. (1983) J. Clin. Chem. Clin. Biochem. 21, 709-720.
25. Haeckel, R., (1984) J. Clin. Chem. Clin. Biochem. 22, 265-270.

26. Staber, G., Busch, E. W. \& Koller, P. U. (1982) Med. Lab. $35,10-13$.

27. Richterich, R. (1965) Klinische Chemie, Theorie und Praxis, 1. Aun., Akademische Verlagsgesellschaft Frankfurt a. $\mathrm{M}$.

28. Bayer, M. F. \& McDougall, J. R. (1982) Clin. Chim. Acta $118,209-218$.

29. Symons, R.G., Walichnowski, Ch. M. \& Murphy, L.J. (1982) Clin. Chem. 28, 266-277.

30. Hopp, T.P. \& Woods, K. R. (1981) Proc. Natl. Acad. Sci. 78, 3824-3828.

31. Burr, W. A., Evans, S. E., Lee, J., Princé, H. P. \& Ramsden, D. B. (1979) Clin. Endocrinol. 11, 333-342.

32. Levy, R.P., Marshall, J.S. \& Velayo, N.L. (1971) J. Clin. Endocrinol. Metab. 32, 372-381.

33. Rudorff, K.-H., Herrmann, J. \& Krüskemper, H. L. (1982), Internistische Welt 3/1982, 102-108.

34. Rudorff, K.-H., Herrmann, J., Dietrich, T. \& Krüskemper, H. L. (1978) Med. Klin. 73, 1109-1113.

35. Rudorff, K.-H. (1979) Fortschr. Med. 97, 2038-2046.

36. Refetoff, S., Fang, V.S., Marshall, J.S. \& Robin, N.J. (1976) J. Clin. Invest. 57, 485-95.

37. Oppenheimer, J.H. \& Werner, S.C. (1966), J. Clin. Endocrinol. Metab. 26, 715-721.

38. Scriba, P. C., Bauer, M., Emmert, D., Fateh-Moghadam, A., Hofmann, 'G. G., Horn, K. \& Pickardt, C. R. (1979) Acta Endocrinol. 91, 629-643.

39. Marshall, J.S. \& Pensky, J. (1969) J. Clin. Invest. 48, 508-515.

40. Burrow, G. N., Polackwich, R., Donabedian, R. (1975) In: Perinatal Thyroid Physiology and Disease (Fisher, D. A. \& Burrow, G. N., eds.) pp. 1-10, Raven Press, New York.

41. Rudorff, K.-H., Schmitz, B. \& Jahnke, K. (1982), Aktuelle Endokrinol. Stoffw. 3, 50-55.

42. Wellby, M. L., Guthrie, L. L. \& Reilly, C.P. (1981) Clin. Chem. 27, 2022-2024.

43. Börner, W., Ruppert, G., Moll, E. \& Reiners, Chr. (1980) Der Nuklearmediziner 3, 235-241.

44. Barlow, J.W., Csicsmann, J.M., White, E. L., Funder, J.W. \& Stockigt, J.R. (1982) J. Clin. Endocrinol. Metab. 55, 244-250.

45. Attwood, E. C. \& Atkin, G. E. (1982) Ann. Clin. Biochem. 19, 101-103.

Anne-Ch. Kessler

Boehringer Mannheim GmbH

Biochemical Research Center

D-8132 Tutzing 
。 